\title{
Physics-Based Image Reconstruction of SiC Grain Boundaries
}

\author{
Amirkoushyar Ziabari ${ }^{1}$, Charles A. Bouman ${ }^{1}$, Jeffrey M. Rickman² and Jeff. P. Simmons ${ }^{3}$ \\ 1. Electrical and Computer Engineering (ECE), Purdue University, West Lafayette, IN, USA. \\ 2. Department of Material Science and Engineering, Lehigh University, Bethlehem, PA, USA. \\ 3. Air Force Research Laboratory, Wright-Patterson AFB, OH, USA.
}

The continuing progress in transmission electron microscopy along with image reconstruction algorithms have led to significant advancement in micro to nanoscale material characterization. As a powerful alternative to conventional techniques such as Filtered Back Projection (FPB), or algebraic SIRT and DART algorithms [1,2], Model Based Image Reconstruction (MBIR) have demonstrated superior performance for 2D/3D image reconstruction of material [3].

While MBIR and other image reconstruction techniques provide high quality reconstructed images, they mainly rely on bizarrely simplistic regularization parameter estimation in their prior model. This prevents the reconstruction techniques from extracting rigorous quantitative information such as actual interfacial properties in grain boundaries from the projection data. In fact, interactions in material follow certain known physical laws and equations, and can be modelled using modern physic-based approaches. These equations can be used as an input to the prior model, and the physic-based models can be leveraged to estimate corresponding parameters. Therefore, by introducing physics of the problem to the prior model and integrating modern physics modelling in the analysis of microscope image data, one can extract quantitative meaningful properties along with high quality 2D/3D images from the projected data.

The goal of this work is to perform reconstruction of polycrystalline microstructures from their electron microscopy data, using the physical models of internal interfaces (i.e., grain boundaries) as prior model into the MBIR framework. We employ atomistic simulation to calculate the excess interfacial energy per unit area and the width for grain boundaries in a model of silicon carbide ( $\mathrm{SiC}$ ). These parameters serve as input to a phase-field model of a microstructure, and the resulting model constitute a prior in the maximum-a-posteriori (MAP) estimate cost function for image reconstruction.

Atomistic simulations of grain-boundaries in SiC using a modified Tersoff potential were performed [4,5]. For concreteness, we focused on twist boundaries at zero temperature and calculated the associated excess grain-boundary energies per unit area, $\sigma$. Figure 1a shows a schematic of the atomic positions above and below the twist boundary. For three (100) twist boundaries of $\Sigma 13, \Sigma 5$, and $\Sigma 9$, the corresponding interfacial energy densities were calculated to be $4.76 \mathrm{~J} / \mathrm{m}^{2}, 5.63 \mathrm{~J} / \mathrm{m}^{2}$ and $5.79 \mathrm{~J} / \mathrm{m}^{2}$, respectively.

In addition, for these boundaries, we also determined the effective boundary width, $w$, by calculating the planar structure factor, $S(\vec{k})$, for a selected Bragg peak, $\vec{k}$. The diminution in peak heights that attends boundary disorder is shown in Figure 1b. From the shape of these curves one can infer $w$ by assuming that an interface is well-described by a hyperbolic tangent profile. We calculated the interface width to be $4 \AA$ for interfacial energy density of $5 \mathrm{~J} / \mathrm{m}^{2}$.

We next established a connection between these atomistic results and a phase-field model of a grain boundary [6,7]. A simulated phantom of grain boundaries with Poisson noise is shown in Figure 1c. The extracted $w$ and $\sigma$ from atomistic simulations are used to obtain coefficients for the phase field model. 
The main goal is to incorporate physics-based phased field model into the MBIR framework to extract quantitative information from image reconstruction of electron microscopy data, which are typically noisy and blurry. For example, in Fig 1c, the interface location at small grains is uncertain due to noise and the proposed framework in this work eliminates that uncertainty. The general maximum-a-posteriori (MAP) cost function that needs to be minimized is as follows:

$$
c(x)=(-\log p(y \mid x, \phi)-\log p(\theta))
$$

Here $\mathrm{y}$ is the measured projected data from the imaging system, and $x$ represent the property of interest from phase field model that needs to be reconstructed. The first term in equation (1) is a measure of closeness between the measured and projected data and can approximated as $\|y-A x\|^{2}$. Here $A$ is the forward projection matrix. The second term in equation (1) is the prior term, which provide prior information on the property of interest. Typically, the prior term is associated with a regularization parameter that needs to be estimated from the modeling. However, in this work, we use phase field free energy functional as the prior model.

The MAP cost function with Allen-Chan prior can be rewritten as follows:

$$
c(\eta, \theta)=\frac{1}{2}\|y-A \eta \theta\|_{\Lambda}^{2}+\sum_{s \in S} f\left(\eta_{s}\right)+\sum_{s \in S} \frac{\kappa}{2}\left\|\nabla \eta_{s}\right\|^{2}
$$

Where $\Lambda$ is the diagonal matrix of inverse noise variance, $s$ are the lattice points, and $\left\|\nabla \eta_{s}\right\|^{2}=$ $\sum_{i=1}^{p}|\nabla \eta|^{2}$. The vector field order parameter $\eta_{s}=\left(\eta_{s, i}\right)_{i=1 \ldots .}$ describes the phase field.

We are working on a phase field regularized method for quantitative reconstruction of images, based in electron microscope images. Results of the state of the art will be given.

\section{References:}

[1] A. C. Kak in Princ. Comput. Tomogr. Imaging (2001), p. 275.

[2] K. J. Batenburg and J. Sijbers, IEEE Trans. Image Process. 20 (2011), p. 2542.

[3] S. Venkatakrishnan et al, in Stat. Signal Process. Work. (2012), p. 2.

[4] N. Goel et al, J. Appl. Phys. 118 (2015), p. 25.

[5] J.-P. Crocombette and L. Gelebart, J. Appl. Phys. 106 (2009), p. 83520.

[6] H. Garcke, B. Nestler, and B. Stoth, Phys. D Nonlinear Phenom. 115 (1998), p. 87.

[7] D. Fan and L.-Q. Chen, Acta Mater. 45 (1997), p. 611.

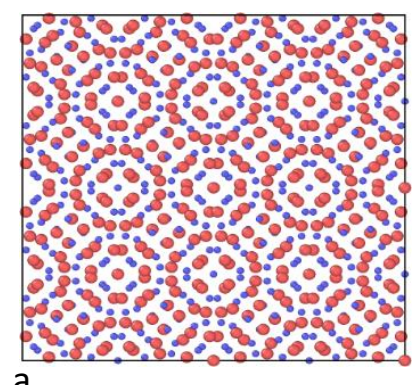

a

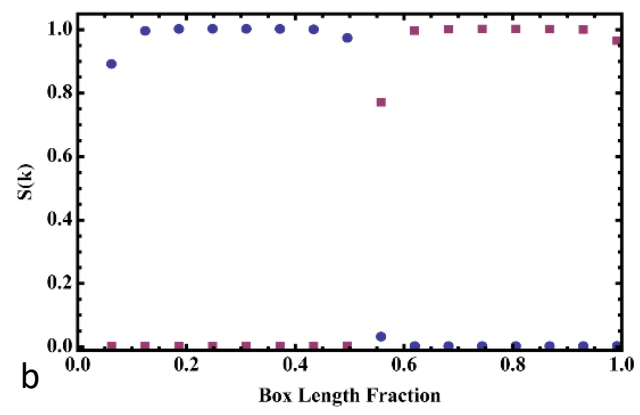

C

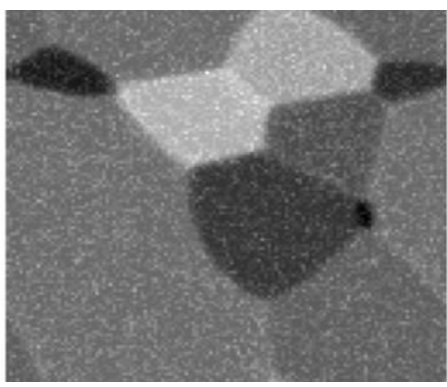

Figure 1. a. The atomic positions at (100) $\Sigma 25$ twist grain boundary in SiC. b. The structure factor plot. c. A simulated phantom of grain boundaries with Poisson noise. 